\title{
VARIABLES E INDICADORES QUE CARACTERIZAN EL APRENDIZAJE-TRABAJO COOPERATIVO EN EL CURSO DE TECNOLOGÍA DE CAFÉ OFRECIDO EN LÍNEA
}

\author{
Campo Elías Riaño Luna ${ }^{8}$
}

RESUMEN

Los resultados de este trabajo sustentan que los estudiantes del curso Tecnología de Café ofertado en línea, asistido con la técnica de aprendizaje basado en problemas, desarrollan la autonomía, la interdependencia, la responsabilidad, la interacción constructiva, la comunicación interpersonal y la evaluación grupal, características importantes del aprendizaje y del trabajo cooperativo. Igualmente se demuestra la aceptabilidad, la pertinencia y la relación que tienen determinados factores demográficos de los estudiantes del curso en comento con las características del aprendizaje cooperativo, concluyendo que es una técnica que estimula el aprendizaje, y los estudiantes lo prefieren al aprendizaje individualista.

Palabras clave: responsabilidad compartida, interacción constructiva, evaluación grupal, aprendizaje cooperativo.

\begin{abstract}
The result of this work supports that the students of the course of Coffee's Technology offered on line, helped with the learning techniques based on problems, develop the autonomy, the interdependence, the responsibility, the constructive interaction, the interpersonal communication and the group evaluation, which are important characteristics of the learning and the cooperative work. Also there is demonstrated the acceptability, the relevancy and the relation that certain demographic factors of the course students have with the characteristics of the cooperative learning, concluding that this is a technique which stimulates the learning and which the students prefer than the individual learning.
\end{abstract}

Key words: shared responsibility, constructive interaction, group evaluation, cooperative learning.

\section{INTRODUCCIÓN}

La Universidad Nacional Abierta y a Distancia UNAD (antes UNISUR), creada por la Ley 52 de 1981, es un establecimiento público, adscrito al Ministerio de Educación Nacional que siempre se ha preocupado por resolver los problemas de la comunidad, en este caso, dar respuesta a problemáticas de tipo educativo-empresarial, objetivo complejo que exige adquirir competencias que posibiliten insertar a la población profesional en actividades

8 Docente UnAD. Grupo de Investigación: ALFUNC-UnAD, Código de registro del grupo en Colciencias: COL0053126, UNAD. 
políticas, sociales, culturales y laborales para las cuales la educación tradicional ya no es suficiente.

Esta es una de las razones por las cuales la UNAD contempla, en el sistema de Gestión de la Calidad, la operación dos: "contribuir al mejoramiento de la prestación de un servicio de alta calidad en la educación a distancia" y la operación tres que recomienda "proporcionar ambientes de aprendizaje autónomo y significativo, para que los estudiantes de educación abierta y a distancia- EAD, desarrollen las competencias exigidas en su formación profesional integral articuladas a las propuestas curriculares" (Leal, Salazar, Ramón y Vidal 2007, p. 191). Dichos objetivos institucionales comprometen a todos y cada uno de los miembros de la comunidad universitaria de la UNAD a diseñar estrategias académicas y organizacionales, para lograr la prestación de un mejor servicio en los cursos que se ofrecen en las Escuelas, en la modalidad a distancia. La acción docente que se desarrolla en línea, se debe fundamentar en el principio de responsabilidad compartida entre los estudiantes y el docente-tutor, toda vez que el centro de los procesos formativos que fomenta la Universidad es el aprendizaje autónomo de sus participantes, haciendo uso de metodologías didácticas con aplicaciones de las TIC.

Acorde con Driscoll, M., (2005, págs. 8, 9, 10) el aprendizaje es un cambio persistente en las transformaciones de las funciones humanas ejecutables y definidas como conducta. Los fenómenos conductuales explicados mediante teorías, conformadas por una serie de constructos enlazados para dar explicación a los cambios observados en el comportamiento de los individuos constituyen las concepciones y tendencias que permiten ubicar el aprendizaje entre dos polos: el cognoscitivismo y el conductismo. Para el cognoscitivismo el papel activo lo tiene el estudiante, que es quien elabora, crea o desarrolla constructos, estructuras, metas o conceptos del mundo que discurre a su alrededor, y considera que el alumno todo lo hace sin una influencia o estímulo externo para aprender.

En cambio en el conductismo, el aprendizaje es visto como un proceso gradual de reforzamiento de respuestas, con incorporación gradual de patrones de conducta existentes. Desde el punto de vista del enfoque histórico-cultural de Vigotsky (1984) (citado por Febles, 1999), el aprendizaje es "un proceso que partiendo de lo externo, del medio como fuente proveedora, se realiza por y en el individuo, atendiendo a sus necesidades a través de la actividad y la comunicación propias, así como de otros, como portadores estos últimos, de toda la riqueza individual y social."

Una de las estrategias pedagógicas para impartir la instrucción es el trabajo en grupo cooperativo, basado en la construcción colectiva del conocimiento, en donde cada miembro del grupo cooperativo es responsable, tanto de su progreso académico como el de los demás integrantes del mismo y donde el tutor es un mediador.

Autores como Ferreiro (2003) son de la opinión de que los ambientes cooperativos en los procesos académicos están orientados y fundamentados en la teoría social y en la conductista, en donde se requiere del conocimiento bien claro del contexto en el cual el sujeto puede aprender o alcanzar la zona de desarrollo próxima, que potencia aprendizajes superiores. 
Autores como Ferreiro (2003) son de la opinión de que los ambientes cooperativos en los procesos académicos están orientados y fundamentados en la teoría social y en la conductista, en donde se requiere del conocimiento bien claro del contexto en el cual el sujeto, puede aprender o alcanzar la zona de desarrollo próxima, que potencia aprendizajes superiores.

A su vez Jorba y Sanmartí (1994), citados por Méndez-Estrada, V. y Monge Nájera, J. (s/f) sostienen que en el aprendizaje cooperativo "Los estudiantes aprenden discutiendo entre ellos y con el profesor o profesora, comparando, desarrollando valores y actitudes más o menos favorables al aprendizaje".

Por lo tanto, el docente que desee implementar la estrategia del aprendizaje cooperativo-AC, apoyado en la pedagogía vigostkyana, debe ser un profundo conocedor de la dinámica de los grupos de estudio y aprendizaje, puesto que en el AC no se trata de hacer una síntesis de contenidos para el logro de aprendizajes consignados por el docente, sino de estimular el compromiso con la cooperación de forma tal que quienes tengan más conocimiento, entiendan más, comprendan más y hayan desarrollado más estrategias de pensamiento para "aprender a aprender" colaboren con los que poseen un nivel de desarrollo inferior y estén interesados en lograr aprendizajes significativos.

Para estimular el trabajo grupal una de las técnicas utilizadas en las aulas es el aprendizaje basado en problemas (ABP). Técnica pedagógica centrada en el estudiante, una forma de trabajo en grupo cooperativo basado en la construcción colectiva del conocimiento y el desarrollo de habilidades mixtas de aprendizaje, desarrollando actitudes básicas como la colaboración, la autonomía y la interacción siendo cada miembro del grupo cooperativo responsable, tanto de su progreso académico como el de los demás integrantes del mismo, donde el profesorado es responsable y mediador.

Pero agrupar varias personas no implica la conformación de un grupo cooperativo, ya que el alumno debe ser activo, participativo, comprometido con su propio aprendizaje y con el de sus compañeros de equipo.

Razón que nos llevó a diseñar y plantear la realización de una encuesta encaminada a determinar si el aprendizaje basado en problemas, es una herramienta que permite el desarrollo de habilidades, competencias y capacidades para el trabajo compartido y la formación integral de los estudiantes del curso de Tecnología de Café.

\section{Metodología empleada}

Como estrategia pedagógica para impartir la instrucción y facilitar el desarrollo de la zona próxima en el curso en comento se plantearon actividades grupales siguiendo la metodología de ABP. Teniendo como referente el trabajo de Johnson, Johnson y Holubec (1999) son cinco las variables a evaluar y analizar en un grupo de trabajo cooperativo: 
La autonomía: porque el estudiante es el responsable de su instrucción y por lo tanto, de la autogestión y autocontrol del proceso de aprendizaje.

La interdependencia: porque el usuario elige qué, cómo y cuándo aprende, debido a la flexibilidad de espacio y tiempo.

La responsabilidad individual y grupal: actitud que existe cuando aquello que ha realizado cada cual, revierte en la totalidad del grupo y de manera individual en cada uno de sus miembros.

La interacción interpersonal y grupal, la cual se realiza a través de diferentes medios tecnológicos, que reducen los obstáculos de espacio y tiempo en el grupo.

Para los fines de la presente investigación, la encuesta la responde individualmente cada estudiante. Los encuestados han venido siendo los estudiantes del curso Tecnología de Café matriculados en los primeros semestres de 2008, 2009 y 2010, por tanto, se recogen tantos cuestionarios como alumnos se hayan entrevistado.

Son estudiantes a distancia y las encuestas se desarrollaron en el campus virtual de la UNAD. Dichos estudiantes cursan la materia a través de la web y previamente han sido entrenados en el manejo eficiente de la plataforma de la Universidad, el navegador, el correo electrónico.

Los estudiantes ingresan al cuestionario siguiendo el enlace: http://www.encuestafacil.com/ MiArea/Crear_Editar_Encuesta.aspx?EID $=455731$

Para la validez del cuestionario se recurrió a la consulta de tres (3) docentes de la UNAD, con experiencia en el área, quienes realizaron algunas observaciones a la redacción, estando de acuerdo con las temáticas evaluadas. De esta forma se puede decir que el cuestionario propuesto presenta validez de contenido, porque las variables analizan las temáticas en estudio. Una vez diligenciado por el estudiante este lo envió y las respuestas dadas fueron almacenadas y analizadas.

Debido a la naturaleza de los datos se utilizaron pruebas, no paramétricas, como Chi cuadrado, para determinar la significancia de las variables y sus indicadores en el estudio y el coeficiente de contingencia de Pearson, para establecer correlaciones. Los datos se procesaron con el paquete estadístico S.P.S.S., versión 15.0 de Windows. (George \& Mallery, 2008).

Cada uno de los informes que se ha venido generando se compone de dos partes; la primera de ellas recoge los datos de opinión de los estudiantes y la segunda debe presentar las frecuencias absolutas observadas para cada ítem-pregunta, teniendo en cuenta para el análisis las frecuencias superiores al $40 \%$. 


\section{RESULTADOS}

Concluido el plazo para diligenciar el formulario, una tabla plana con todos los registros recolectados fue exportada a una carpeta asignada para este fin y los valores fueron llevados al editor de datos del programa estadístico SPSS versión 15 obteniéndose, entre otras, la siguiente información:

Tabla 1. Resumen de los principales datos del proceso de encuesta aplicado a estudiantes.

\begin{tabular}{|l|r|}
\hline Población (estudiantes matriculados)/curso & 165 \\
\hline Error muestral para el total de la población $(95 \%$ de Nivel de confianza, $\mathrm{p}=\mathrm{q}=0.5$ & $0.5 \%$ \\
\hline Genero que más participo & Masculino \\
\hline Nivel académico de mayor participación & Tecnólogo \\
\hline Moda de la edad & {$[21-30]$} \\
\hline Moda de estado civil & Solteros \\
\hline Moda de escuela & ECBTI \\
\hline Estadístico de fiabilidad- Alfa de Cronbach para los 35 preguntas de la encuesta & 0,908 \\
\hline
\end{tabular}

Fuente: el autor

\section{CONCLUSIONES}

Los resultados constituyen una parte de la evaluación de las actividades desarrolladas por los estudiantes, como establece la normativa institucional de la UNAD, en el Artículo 32, evaluación del rendimiento académico. Leal, Salazar, Ramón y Vidal (2007, p.99).

Mediante el análisis descriptivo se pueden analizar los diferentes ítems de la encuesta aplicada, en forma individual y en línea, a los estudiantes matriculados en el curso en comento.

Los datos obtenidos permiten estimar para una significancia de $1 \%$, varios grados de correlación entre las variables demográficas y las variables cooperativas en estudio.

Del análisis realizado se infiere que la mayoría de los estudiantes manifiesta que los grupos colaborativos están enmarcados dentro de los parámetros exigidos por la Universidad para lograr una mejor atención al estudiante, facilitando su aprendizaje.

Acorde con George \& Mallery (2008, P.251), cuando el valor de alfa para determinar la fiabilidad es mayor de 0.9 los datos sobre los cuales se calcula son excelentes y confiables. Por lo tanto, la herramienta trabajada en esta investigación permite evaluar cada uno de las variables e indicadores que caracterizan el trabajo cooperativo en el curso de Tecnología de Café. 


\section{RECOMENDACIONES}

Por ser una de las primeras investigaciones realizadas en este ámbito, en el curso de Tecnología de Café de la UNAD, los resultados no se pueden considerar definitivos, por lo que se sugiere realizar un estudio más en detalle.

\section{REFERENCIAS BIBLIOGRÁFICAS}

DRISCOLL, M. (2005), Psychology of Learning for Instruction (3ra ed.). Needham Heights, MA: Allyn and Bacon.

FEBLES, M. (1999), Un punto de vista sobre el carácter activo del sujeto del aprendizaje. En Revista Cubana de Psicología, Vol. 16, No. 3, pp. 214-221.

FERREIRO, R. (2003), Estrategias didácticas del aprendizaje cooperativo. México. Trillas.

FERREIRO, R. (2007), Aprendizaje cooperativo. Revista Electrónica de Investigación Educativa, 9 (2). Disponible en: http://redie.uabc.mx/vol9no2/contenido-ferreiro.html Consultado el 09/julio/09.

GALL, M. D., GALL, J., Y BORG, W. (2006), Educational research: An introduction (8va. ed.). Boston: Pearson Education.

JOHNSON, D., JOHNSON, R., HOLUBEC, E, (1999): El aprendizaje cooperativo en el aula. Buenos Aires: Paidós.

LEAL, J., SALAZAR,R., RAMÓN, M.\& VIDAL, E, (2007). Inducción unadista: Educacion para todos. Bogotá, Colombia: UNAD.

MÉNDEZ-ESTRADA, V. Y MONGE NÁJERA, J. (s/f), Las TIC en un entorno latinoamericano de educación a distancia: la experiencia de la UNED de Costa Rica. RED. Revista de Educación a Distancia. Página 7 de 13. Consultado el 10 de julio de 2009 en http://www.um.es/ead/red/15 